\title{
Use of sedatives and muscle relaxants in newborn babies receiving mechanical ventilation
}

\author{
Malcolm I Levene, Michael W Quinn
}

In the 1970 s sedation or paralysis was rarely considered to be necessary in the ventilatory care of premature infants. We now recognise that infants who 'fight' the ventilator may be at increased risk of a number of important neonatal complications including intracranial haemorrhage and pneumothorax. There is no consensus view on the best way of managing these infants, nor what pharmacological preparations to use in reducing the risks of these complications. When formulating an approach to inhibiting spontaneous respiration in the ventilated neonate there are three important issues to be considered. These are (i) what are the hazards of the infant breathing out of synchrony with the ventilator?, (ii) what are the effects of stress and pain associated with mechanical ventilation?, and (iii) what are the possible adverse effects of drugs used to reduce struggling or stress?

\section{The baby and the ventilator}

Anyone involved in mechanical ventilation of infants will recognise the infant who 'fights' the ventilator. Defining this is somewhat more difficult. Greenough et al identified infants who were actively expiring at the time of ventilator inflation as being particularly at risk of developing pneumothorax. ${ }^{1}$ They used an intraoesophageal balloon to detect spontaneous respiratory activity and a pneumotachograph to measure tidal flow. This system is neither available nor practicable in most newborn units and other methods to recognise this pattern have been adopted. The simplest is observing the baby's respiratory activity in relation to the mechanical ventilator. Although this may successfully identify some babies, particularly those who are vigorously breathing (usually mature newborn infants) or those who are mechanically ventilated at slow rates, the observer may be in some doubt as to the interaction between baby and machine. An alternative method is to examine the beatto-beat variability on an undamped arterial blood pressure trace. When an infant breaths out of phase with the ventilator there is increased fluctuation between the maximum and minimum systolic peaks, and if the coefficient of variation exceeds $10 \%$ we believe that this represents 'fighting'. In practice, observation alone of the blood pressure trace confirms the clinical impression of the baby showing this pattern.
Perlman et al showed that ventilated infants who had a fluctuating pattern of cerebral blood flow velocity (CBFV) in the anterior cerebral artery were at increased risk of subsequently developing intraventricular haemorrhage $(\mathrm{IVH}) .^{2}$ This fluctuating pattern directly reflected fluctuating arterial blood pressure as discussed above. They suggested that interventions to reduce fluctuating CBFV may reduce the incidence of IVH.

We assume that intensive care is an abnormally stressful event for newborn infants. There are many interrelated components of stress which are not understood and have not yet been unravelled.

It is likely that 'fighting' the ventilator is one factor in the stress situation. Adults who have been pharmacologically paralysed to facilitate mechanical ventilation and who become aware describe it as being a very frightening experience. This may be compounded by painful procedures undertaken on the paralysed, but aware, patient. It is not unreasonable to believe that the newborn may be similarly stressed by neuromuscular paralysis in an 'awake' state.

\section{Muscle relaxants}

Pancuronium is a steroidal non-depolarising muscle relaxant and was the first to become widely used in clinical practice. It is metabolised in the liver and the metabolites are excreted mainly in the urine. Because of the dependence of this drug on liver metabolism and renal excretion, the length of action will be prolonged in premature infants with immature renal and hepatic function. Infants and children are more sensitive to the drug than adults and take longer to clear it. ${ }^{3}$

CLINICAL ROLE OF PANCURONIUM

Perlman et al showed that muscle paralysis of babies with a fluctuating CBFV pattern led to a significant reduction in the incidence of IVH. ${ }^{4}$ Those paralysed infants who developed IVH did so after the cessation of paralysis. In a larger randomised case control study of unselected babies, Bancalari et al (abstract, Pediatric Res 1980; 14: A986) failed to demonstrate a protective effect of pancuronium in IVH. The infants who were paralysed actually had a significantly higher incidence of massive IVH. We have similarly failed to find a reduction in IVH in paralysed infants. 
Greenough et al selectively paralysed infants with pancuronium who actively expired against positive pressure inspiration. ${ }^{1}$ All the infants who were not paralysed developed a pneumothorax while only one of the 11 who were paralysed did so. The authors suggested that muscle paralysis should only be used in selected patients in view of the psychological problems for patients associated with use of paralysing agents'. Cooke and Rennie reviewed the incidence of pneumothorax in their unit before and after the use of muscle paralysis for ventilated newborn infants who struggled against the ventilator. ${ }^{5}$ They showed a reduction in the incidence of pneumothorax in infants with a gestation age $\geq 27$ weeks when pancuronium was used. Bancalari, found no reduction in the incidence of pneumothorax in a large randomised study of all ventilated newborn infants. Pollitzer et al similarly found no reduction in the rate of pneumothorax in an unselected group of ventilated infants who were paralysed. ${ }^{6}$ It is not possible to agree on the clinical indication for pancuronium in the prevention of pneumothorax, but there appears to be good evidence that it does prevent pneumothorax in selected cases. Unfortunately, the criteria for this selection are difficult to apply on a regular basis in a busy neonatal unit.

Pollitzer et al have shown a significant reduction in the incidence of chronic lung disease in ventilated infants who were paralysed. ${ }^{6}$ All ventilated infants with hyaline membrane disease were entered into this trial, with no selection criteria. A recent study has shown an increase in pulmonary resistance and reduction in dynamic pulmonary compliance in paralysed infants. ${ }^{7}$ This was not a controlled study and the changes may have been due to natural progression of the disease process. None the less, there was a close temporal association between starting and discontinuing pancuronium, and deterioration and improvement in pulmonary function tests respectively. A suggested explanation for this was a loss of negative intrapleural pressure leading to impairment of drainage of lung fluid.

It may not be necessary to use any drugs to improve synchrony with the ventilator. The Cambridge group have shown that careful alterations in ventilator settings may allow the ventilator to 'capture' the infant's own respiratory efforts. ${ }^{8}$ This in practice is time consuming and is not likely to be practical in the setting of junior medical staff making most of the ventilator adjustments in a busy neonatal unit.

\section{POTENTIAL SIDE EFFECTS}

Pancuronium causes complete loss of muscle power and ventilatory effort. If this is not anticipated, severe hypoxia may result from inadequate chest wall movement. Studies suggest that, although the acute effects of pancuronium on oxygenation are unpredictable, it does appear to reduce the variability in gas exchange over a more prolonged period. ${ }^{9}$

The cardiovascular effects of pancuronium are variable. Pancuronium should not alter blood pressure as ganglion blockade and histamine release are negligible. One case report suggested that pancuronium could cause severe hypotension, ${ }^{10}$ but clinical studies of its effect on blood pressure and heart rate have shown no consistent trend. ${ }^{11}$

A commonly observed effect of long term pancuronium administration is that infants become oedematous. Greenough et al found that paralysed infants regained their birth weight sooner than non-paralysed controls despite fluid restriction. ${ }^{12}$ A suggested mechanism was hypoperfusion of the kidneys resulting in relative oliguria. This was supported by the higher urinary osmolarity in the paralysed infants. Long term paralysis may lead to chest wall oedema and impaired compliance. The development of joint contractures has also been reported.

\section{Drugs for sedation and analgesia}

Another approach is to inhibit the baby's respiratory drive with opioid drugs. Morphine has been the most widely used in this context. Understanding the metabolism and disposition of these drugs is essential if side effects are to be avoided. These include hypotension, reduced gut motility, muscle rigidity, and fits.

Morphine is metabolised in the liver by glucuronidation and sulphation with the products being excreted in the urine. ${ }^{13}$ Morphine3-glucuronide (M3G) is an inactive metabolite but there is evidence that morphine-6glucuronide (M6G) is a more potent analgesic than morphine itself and may have a more profound respiratory depressant effect. ${ }^{14}$ It is often present in plasma in greater amounts than the parent morphine molecule. The analgesic properties of morphine may be largely dependent on the rate of conversion to M6G. Both the rate of clearance of morphine and the rate of metabolism to $\mathrm{M} 6 \mathrm{G}$ are impaired in neonates. Therefore, higher levels of morphine are likely to accumulate but conversion to M6G will be less efficient. M6G is excreted by the kidneys and poor renal function may lead to accumulation of this metabolite in premature newborn infants. It is unclear whether these factors would result in enhanced or impaired analgesic activity of morphine in the newborn.

\section{CLINICAL ROLE OF OPIATES}

There is anecdotal evidence that the newborn infant is more sensitive to the respiratory depressant properties of morphine. ${ }^{15}$ This may be related to impaired metabolism and/or increased penetration into the brain. There is little published data on the effectiveness of morphine in improving synchrony in ventilated neonates but our clinical impression has been that morphine, even in high doses, may not suppress the infant's ventilatory effort sufficiently to improve synchrony.

Diamorphine, which is metabolised to morphine, has recently been carefully evaluated in the newborn infant. ${ }^{16}$ It has a rapid onset of action and is more potent than morphine. A 
dose regimen at the lower end of that for morphine was used because of its higher potency. A bolus dose of $50 \mu \mathrm{g} / \mathrm{kg}$ was followed by an infusion rate of $15 \mu \mathrm{g} / \mathrm{kg} /$ hour. There was a positive correlation between clearance and gestational age. Careful monitoring of blood pressure showed a small but significant fall and there was reduction in respiratory rate which was taken to indicate improved synchrony with the ventilator.

Fentanyl and alfentanil are synthetic opioid analgesics which are considerably more potent than morphine. They have the theoretical advantages of shorter duration of action and greater cardiovascular stability because of less histamine release. These advantages have made them attractive for use in the neonatal period, particularly if the shorter duration of action allows for quicker weaning from the ventilator. Marlow et al used a loading dose of alfentanil of $15-20 \mu \mathrm{g} / \mathrm{kg}$ followed by infusion of 3-5 $\mu \mathrm{g} / \mathrm{kg} /$ hour in ventilated preterm infants. ${ }^{17}$ These infants had reduced clearance and a prolonged elimination half life when compared with older children and adults but there was marked variability in metabolism between individuals.

\section{ANALGESIA}

The measurement of analgesic efficacy is difficult in the ventilated premature newborn. There is some evidence from pain studies in infants that the concentration of morphine required to produce analgesia is higher than in older children. The reason for this is not clear. There is no information on analgesic concentrations of morphine in premature neonates. Even if these data were available, the variation in the metabolism of morphine and the analgesic effect of its metabolites would make the accurate determination of an appropriate dosage regimen difficult.

Pain in the newborn infant may be measured using behavioural, physiological, and endocrine responses. It is difficult to assess behavioural responses in an intubated infant and physiological responses may be difficult to interpret in a sick infant. Humoral and endocrine markers may provide useful information. Anand et al demonstrated a marked endocrine response in infants undergoing surgery for patent ductus arteriosus who had not received adequate analgesia. ${ }^{18}$ There was an increase in adrenaline, noradrenaline, and glucagon, with a reduction in insulin concentrations. These changes were ablated by preoperative treatment with fentanyl. There was also evidence that those who received fentanyl had fewer postoperative complications. The same workers had earlier shown a graded endocrine response depending on whether the infants where subjected to mild, moderate, or severe surgical stress.

We have recently reported a randomised study comparing the effects of morphine alone (50-100 $\mathrm{\mu g} / \mathrm{kg} /$ hour by infusion), pancuronium alone (100 $\mu \mathrm{g} / \mathrm{kg} /$ dose, as required), and a combination of morphine and pancuronium in reducing catecholamine concentrations in struggling ventilated neonates (abstract pre- sented at the British Paediatric Association meeting, Warwick 1991.) Those who received morphine showed a significant reduction in noradrenaline concentrations while those who received pancuronium alone or pancuronium with morphine showed no such reduction. The reduction in noradrenaline concentrations was modest and much less than those reported in Anand's moderate and severe surgical stress groups. A number of infants were withdrawn from the morphine arm of the trial because the infants continued to struggle, probably because no loading dose was used. We concluded that infants who settle on morphine show a significant reduction in stress hormone concentrations.

Although it is not known whether intubation and ventilation are painful for premature newborn infants, behavioural clues that an infant feels pain, such as withdrawal and grimacing, are lost in paralysed infants. It may then be forgotten that procedures undertaken in paralysed but 'awake' infants are painful. This may be an additional important reason for giving continuous analgesic medication to paralysed infants.

\section{POTENTIAL SIDE EFFECTS}

Although, theoretically, opiates may prolong the length of time spent on the ventilator, we found no significant difference in the number of ventilator days between morphine and pancuronium treated infants in our randomised trial.

Hypotension is a serious potential complication of morphine. We have carefully monitored infants for this complication and found no reduction in blood pressure six hours after starting morphine by continuous infusion. Continuous blood pressure monitoring in a group of eight infants who received a bolus dose of morphine of $400 \mu \mathrm{g} / \mathrm{kg}$ over two hours showed no evidence of hypotension. This may be because the bolus dose was given over a prolonged period. Marlow et al ${ }^{17}$ found a transient but significant reduction in blood pressure when a bolus of alfentanil was given over two minutes. The rate of drug administration is probably important.

Muscle rigidity is a reported complication of opiate administration. ${ }^{19}$ Despite careful observation over the last few years we have only observed this effect transiently in two infants. Fits after morphine have been described in two neonates who were on standard rates of morphine infusion (32 and $40 \mu \mathrm{g} / \mathrm{kg} / \mathrm{hour}$ ) and the drug concentrations were not high $(61$ and $90 \mu \mathrm{g} / 1){ }^{20}$ Similar concentrations have been reported without the occurrence of seizures. This may have been an idiosyncratic response and has not been subsequently reported.

Withdrawal symptoms may occur after prolonged administration of any opiate, but these are unlikely after short courses usually given to ventilated neonates. We have not recognised withdrawal symptoms in a newborn infant given morphine for therapeutic indications. A recent study has shown that withdrawal symptoms are much more common 
after fentanyl than morphine. ${ }^{21}$ The symptom score was that used to assess term infants of narcotic addicted mothers. This is an entirely different group of infants on which it may not be appropriate to use the same symptom score. This may explain the high incidence of withdrawal symptoms in both groups despite careful tapering of the dose.

The endogenous opiate receptors appear to be functional in the immature nervous system and we do not know whether there may be long term effects of high concentration infusion of exogenous opiates such as morphine. The functional integrity of the endogenous opiate system must be further studied for long term effects after cessation of the infusion.

\section{Current practice}

The evidence that muscle paralysis prevents IVH is poor and its role in preventing pneumothorax is uncertain. At present we use pancuronium sparingly. The indications include mature babies (>34 weeks) who make vigorous respiratory efforts and whose ventilatory efforts are clearly detrimental to gas exchange. This decision is made clinically depending on how much the infant's respiratory effort is out of synchrony with the ventilator and the variability of the arterial blood pressure trace. Paralysis is frequently employed after thoracic surgery where an anastomosis may be torn if the infant struggles (for example, a repair of oesophageal atresia). If a paralysed infant is likely to need long term ventilation or be subjected to painful procedures, then a simultaneous morphine infusion is continued for at least four days.

We feel that infants with hyaline membrane disease who have received exogenous surfactant (Curosurf, Chiesi Pharmaceuticals, in our unit) 'fight' the ventilator to a considerably lesser extent. There is often a rapid fall in the baby's oxygen requirements after the administration of this type of surfactant, probably reflecting the change in lung compliance.

We consider that 'awake' intubation and ventilation exacerbates the infant's stress responses and therefore we start on morphine every infant who is likely to need ventilation for more than a few hours. A combination of bolus followed by infusion will ensure that an adequate plasma concentration is reached quickly and maintained. We administer the bolus dose over two hours to reduce the risk of adverse effects. From published data on volume of distribution and clearance, we have calculated a dosage regimen that aims to achieve a plasma concentration of $100 \mu \mathrm{g} / \mathrm{l}$ at steady state. ${ }^{22}$ This is higher than the level shown to be analgesic in older children $(65 \mu \mathrm{g} / 1)^{23}$ and aims to produce respiratory depression. Because of wide interindividual variation, this figure can only be an approximation. Our present protocol is to give
$100 \mu \mathrm{g} / \mathrm{kg} /$ hour for two hours followed by a continuous infusion of $25 \mu \mathrm{g} / \mathrm{kg} /$ hour. We continue the infusion until the inspired oxygen concentration is less than $40 \%$. Because this should achieve adequate analgesic concentrations quickly, we feel that the baby is protected from repeated painful procedures such as venepuncture and heel stabs. Insertion of chest drains are, however, likely to be extremely painful and we do recommend that local anaesthetic is infiltrated superficially and into the deep tissues before insertion of the drain.

1 Greenough A, Morley CJ, Wood S, Davis JA. Pancuronium prevents pneumothoraces in ventilated premature babies who actively expire against positive pressure inflation. Lancet 1984;i: 1-3.

2 Perlman JM, McMeranin JB, Volpe J. Fluctuating cerebral blood-flow velocity in respiratory-distress syndromerelation to the development of intraventricular haemorrhage. N Engl I Med 1983;309:204-9.

3 Smith CE, Baxter M, Bevan JC, Panati F, Bevan DR. Accelerated onset and delayed recovery of $d$-tubocurarine blockade with pancuroniu

4 Perlman JM, Goodman S, Kreusser KL, Volpe J. Reduction in intraventricular haemorrhage by eliminaReduction in intraventricular haemorrhage by elimina-
tion of fluctuating cerebral blood flow velocity in preterm infants with respiratory distress syndrome. preterm infants with respirato

5 Cooke RWI, Rennie JM. Pancuronium and pneumothorax. Lancet 1984;i:286-7.

6 Pollitzer MJ, Shaw DG, Reynolds EOR, Thomas RM. Pancuronium during mechanical ventilation speeds recovery of lungs of infants with hyaline membrane disease. Lancet 1981;i:346-8.

7 Bhutani VK, Abbasi S, Sivilri EM. Continuous skeletal muscle paralysis: effect on neonatal pulmonary mechanics. Pediatrics 1988;88:419-22.

8 South M, Morley CJ. Synchronous mechanical ventilation of the neonate. Arch Dis Child 1986;61:1190-5.

9 Finer NN, Tanney PM. Controlled evaluation of muscle relaxation in the ventilated neonate. Pediatrics relaxation in

10 McIntosh $\mathrm{N}$. Hypotension associated with pancuronium use in the newborn. Lancet 1985;ii:279.

11 Runkle B, Bancalari E. Acute cardiopulmonary effects of pancuronium bromide in mechanically ventilated newpancuronium bromide in mechanically
born infants. $\mathcal{F}$ Pediatr $1984 ; 104: 614-7$.

12 Greenough A, Gamsu HR, Greenall F. Investigation of the effects of paralysis by pancuronium on heart rate variability, blood pressure and blood balance. Acta Paediatr Scand 1989;78:829-34.

13 Choonara IA, McKay P, Hain R, Rane A. Morphine metabolism in children. Br F Clin Pharmacol 1989;28:599-604.

14 Osborne R, Joel S, Trew D, Slevin M. Analgesic activity of morphine-6-glucuronide. Lancet 1988;i:828.

15 Way WL, Costley EC, Way EL. Respiratory sensitivity of the newborn infant to meperidine and morphine. Clin Pharmacol Ther 1965;6:454-61.

16 Elias-Jones AC, Barrett DA, Rutter N, Shaw PN, Davis SS. Diamorphine infusion in the preterm neonate. Arch Dis Child 1991;66:1155-7.

17 Marlow N, Weindling AM, Van Peer A, HeyKants J. Alfentanil pharmacokinetics in preterm infants. Arch Dis Child 1990;65:349-51.

18 Anand KJS, Sippell WG, Aynsley-Green A. Randomised trial of fentanyl anaesthesia in preterm babies undergoing surgery: effects on the stress response. Lancet 1987;i:243-8.

19 Askgaard B, Nilsson T, Ibler M. Muscle tone under fentanyl-nitrous oxide anaesthesia measured with a transducer apparatus in choloecystectomy incisions. Acta Anaesthesiol Scand 1977;21:1-4.

20 Koren G, Butt W, Chinyanga H, Soldin S, Tan YK, Pape $K$. Postoperative morphine infusion in newborn infants: assessment of disposition characteristics and safety. $\Im$ Pediatr 1985;107:963-7.

21 Norton JJ. After effects of morphine and fentanyl analgesia:

22 Bhat $R$, Chari G, Gulati A, Aldana O, Velanati R, Bhargava $\mathbf{H}$. Pharmacokinetics of a single dose of morphine in preterm infants during the first week of life. P Pediatr 1990;117:477-81.

23 Dalstrom B, Bolne P, Feychting H, Noack G, Paalzow L. Morphine kinetics in children. Clin Pharmacol Ther 1979;26:354. 